\title{
Acceleration and radiation of ultra-high energy protons in galaxy clusters
}

\author{
G. Vannoni ${ }^{1, \star}$, F. A. Aharonian ${ }^{2,3}$, S. Gabici ${ }^{2,5}$, S. R. Kelner ${ }^{3,4}$, and A. Prosekin ${ }^{3,4}$ \\ 1 Commissariat à l'Énergie Atomique, DSM/Irfu/SPP, 91191 Gif-sur-Yvette, France \\ 2 Dublin Institute for Advanced Studies, 31 Fitzwilliam Place, Dublin 2, Ireland \\ 3 Max-Planck-Institut für Kernphysik, Saupfercheckweg 1, Heidelberg 69117, Germany \\ e-mail: felix.aharonian@mpi-hd.mpg.de \\ ${ }_{4}$ Moscow Engineering Physics Institute, Kashirskoe sh. 31, Moscow 115409, Russia \\ 5 Astroparticule et Cosmologie (APC), CNRS, Université Paris 7 Denis Diderot, 10 rue Alice Domon et Léonie Duquet, \\ 75205 Paris Cedex 13, France
}

Received 29 October 2009 / Accepted 16 September 2011

\section{ABSTRACT}

\begin{abstract}
Context. Clusters of galaxies are believed to be capable of accelerating protons at accretion shocks to energies exceeding $10^{18} \mathrm{eV}$. At these energies, the losses caused by interactions of cosmic rays with photons of the cosmic microwave background radiation (CMBR) begin to have an effect and determine the maximum energy of protons and the shape of the energy spectrum in the cutoff region.

Aims. The aim of this work is to study both the formation of the energy spectrum of accelerated protons at accretion shocks of galaxy clusters and the characteristics of their broad band emission.

Methods. The proton energy distribution is calculated self-consistently via a time-dependent numerical treatment of the shock acceleration process, which takes the proton energy losses due to interactions with the CMBR into account. We calculate the energy distribution of accelerated protons, as well as the flux of broad-band emission produced by secondary electrons and positrons via synchrotron and inverse Compton scattering processes.

Results. We find that the downstream and upstream regions contribute almost at the same level to the emission. For the typical parameters characterising galaxy clusters, the synchrotron and IC peaks in the spectral energy distributions appear at comparable flux levels.

Conclusions. For an efficient acceleration, the expected emission components in the X-ray and gamma-ray bands are close to the detection threshold of current generation instruments, and will possibly be detected with the future generation of detectors. Remarkably, the acceleration of iron at these shocks can proceed up to a particle energy of $\approx 10^{20} \mathrm{eV}$, making clusters of galaxies possible contributors to the observed flux of ultra high energy cosmic rays
\end{abstract}

Key words. acceleration of particles - shock waves - radiation mechanisms: non-thermal - galaxies: clusters: general

\section{Introduction}

Rich clusters of galaxies are the largest virialised structures in the Universe, with typical sizes of a few Mpc and masses up to $10^{15} M_{\odot}$ or more (see Sarazin 1988 for a review). In the standard picture of cosmic structure formation, the structure's growth is driven by gravitational instability. This process is hierarchical, with larger systems forming later via the assembly of pre-existing smaller structures. Within this scenario, galaxy clusters form from mergers, and their age can be estimated to be of the order of 10 Gyr (e.g. Borgani \& Guzzo 2001). In addition, cold material from the surrounding environment is continuously infalling, owing to gravitational attraction, and an expanding shock wave, called the accretion shock, is expected to form at the cluster boundary and to carry the information of virialisation outward (Bertschinger 1985). Numerical simulations have confirmed the appearance of the so-called accretion shocks during structure formation (e.g. Kang et al. 1994).

The detection of a tenuous and diffuse synchrotron radio emission from about one third of the rich clusters of galaxies (e.g. Govoni \& Feretti 2004) reveals the presence of a diffuse

^ Work performed as IMPRS fellow at the Max-Planck-Institut für Kernphysik, Heidelberg. magnetic field and a population of high energy electrons. Using exclusively synchrotron data, though, it is possible to get information only on the product of particles density and magnetic field energy density (unless adopting assumptions like equipartition). Detection of non-thermal X-rays has also been claimed for a few of such sources and generally interpreted as inverse Compton emission from the same population of electrons (e.g. Fusco-Femiano et al. 1999; Eckert et al. 2008). Since IC depends on the electron density but not on the magnetic field, combining the data allows us to break the degeneracy and thus determine values of $B$ of the order of $0.1 \mu \mathrm{G}$. Significantly higher values, of a few microGauss, are obtained from Faraday rotation measurements (Clarke et al. 2001; Carilli \& Taylor 2002). Thus the uncertainty in determining the magnetic field is quite large, allowing values to vary by about one order of magnitude (Newman et al. 2002).

According to theoretical models, the electrons responsible for the radio and non-thermal X-ray emission are produced through different acceleration mechanisms (e.g. Schlickeiser et al. 1987; Tribble 1993; Sarazin 1999; Brunetti et al. 2001; Petrosian 2001). Alternatively, the radio synchrotron radiation can be emitted by secondary electrons produced by the interactions of accelerated protons with the intracluster gas 
(Dennison 1980; Blasi \& Colafrancesco 1999; Atoyan \& Völk 2000; Dolag \& Enßling 2000; Kushnir et al. 2009). In this scenario, gamma-ray emission is expected from the decay of neutral pions produced in $p$ - $p$ interactions (Völk et al. 1996; Berezinsky et al. 1997). The non-thermal X-rays emission also can be related to the synchrotron radiation of secondary electrons of much higher energies produced in photon-photon (Timokhin et al. 2004) and proton-photon (Aharonian 2002; Inoue et al. 2005) interactions.

Several particle acceleration mechanisms have been proposed as operating in clusters of galaxies (see Blasi et al. 2007 for a review). In particular, it has been argued that large-scale shocks can effectively accelerate electrons and protons up to ultrarelativistic energies (Norman et al. 1995; Berezinsky et al. 1997; Loeb \& Waxman 2000; Miniati et al. 2001; Blasi 2001; Gabici \& Blasi 2003; Ryu et al. 2003; Berrington \& Dermer 2003; Pfrommer et al. 2006). In a recent paper we performed detailed calculations of diffusive shock acceleration of electrons in galaxy clusters (Vannoni et al. 2009). Electrons can be accelerated up to $100 \mathrm{TeV}$ at cluster accretion shocks, with synchrotron $\mathrm{X}$-ray and IC gamma-ray radiation components produced mainly in the downstream region. While the maximum energy of electrons is limited by synchrotron and IC energy losses, the protons can be accelerated to much higher energies.

Indeed, according to the Hillas criterion (Hillas 1984), galaxy clusters are amongst the few source populations capable, as long as this concerns the dimensions of the structure and the value of the magnetic field, of accelerating protons up to $10^{20} \mathrm{eV}$. Moreover, clusters are cosmological structures, and their lifetime is comparable to the age of the Universe; therefore, if acceleration takes place in such objects, it can continue up to $\sim 10^{10} \mathrm{yr}$. On the other hand, high-energy protons up to energies of the order of $10^{15} \mathrm{eV}$ and possibly higher are confined well in the volume of the cluster on this time scale (Völk et al. 1996; Berezinsky et al. 1997). This results in an effective accumulation of high-energy particles in the cluster. The thermal energy budget of rich clusters, estimated from measurements of their thermal X-ray emission, ranges from $10^{63}$ to $10^{64} \mathrm{erg}$. The non-thermal component seems to be constrained to a few percent of the thermal energy $\sim 10^{62} \mathrm{erg}$ (Perkins et al. 2006; Aharonian et al. 2009; Aleksic et al. 2010). This value is also comparable to the magnetic field energy, assuming $1 \mu \mathrm{G}$ and a spherical cluster of $3 \mathrm{Mpc}$ of radius (e.g. the size of the Coma cluster).

Although the dimensions of the system, the strength of the magnetic field, and the age of the accelerator formally allow protons to be accelerated up to $10^{20} \mathrm{eV}$, the particles, in fact, lose their energy from pair and pion production in the interactions with the photons of the cosmic microwave background radiation (CMBR) field. As discussed in Norman et al. (1995) and Kang et al. (1997), for a shock velocity of a few thousand $\mathrm{km} \mathrm{s}^{-1}$ and a magnetic field of the order of $1 \mu \mathrm{G}$, the shock acceleration rate is compensated by the energy loss rate at energies around $10^{19} \mathrm{eV}$. The exact value depends on the assumed diffusion coefficient and also on the shock geometry as shown by Ostrowski \& Siemieniec-Ozieblo (2002). The interactions of ultra-high energy protons with the CMBR lead to production of electrons in the energy domain $\left(\geq 10^{15} \mathrm{eV}\right)$, which is not accessible through any direct acceleration mechanism. These electrons cool via synchrotron radiation and IC scattering on very short timescales (compared to both the age of the source and interaction timescales of protons). For the same reason, they are rather localised in space, i.e. are "burned" not far from the sites of their production. Therefore the corresponding radiation components in the X-ray and gamma-ray energy bands are precise tracers of primary protons, containing information about the acceleration and propagation of their "grandparents".

This interesting feature has been indicated in Aharonian (2002) in the general context of acceleration and propagation of ultra-high energy protons in large scale extragalactic structures. More specifically, this issue was discussed by Inoue et al. (2005) for objects like the Coma galaxy cluster. However, in these studies an a priori spectrum of protons has been assumed in the "standard form" $E^{-2} \exp \left(-E / E^{*}\right)$. In fact, the energy distribution of protons accelerated in galaxy clusters via diffusive shock acceleration (DSA) mechanism can be quite different from this simple form, as demonstrated below.

In this paper we study the process of proton acceleration by accretion shocks in galaxy clusters. Energy losses of protons due to their interactions with the CMBR photons are selfconsistently taken into account. We make use of the numerical approach presented in Vannoni et al. (2009). In Sect. 2 the calculation is introduced and the accelerated proton spectra are derived. In particular, we show that electron/positron pair production is the dominant energy-loss channel for protons. In Sect. 3 we calculate the spectra of secondary pairs. The broad-band emission produced by the secondary electrons during their interactions with the background magnetic and radiation fields is presented in Sect. 4. In Sect. 5 we study the impact of shock modification by efficiently accelerated protons on the acceleration and emission features. We briefly discuss and summarise the main results in Sect. 6 .

\section{Proton acceleration and energy losses}

The accretion of cold external material onto a hot rich cluster of galaxies can lead to the formation of a strong shock at the position of the virial radius of the cluster. The shock velocity can be estimated from the free fall velocity of the infalling matter crossing the shock surface:

$v_{s} \sim \sqrt{\frac{2 G M_{\mathrm{cl}}}{R_{\mathrm{cl}}}} \approx 2000\left(\frac{M_{\mathrm{cl}}}{10^{15} M_{\odot}}\right)^{1 / 2}\left(\frac{R_{c l}}{3 \mathrm{Mpc}}\right)^{-1 / 2} \mathrm{~km} \mathrm{~s}^{-1}$.

It is interesting to note that the shock velocities for this case are comparable to the shock speeds typical of young supernova remnants.

We approximate the spherical shock locally as a plane and work in the reference frame where the shock is at rest and the plasma moves along the $x$-axis, perpendicular to the shock surface, from $-\infty$ far upstream, to $+\infty$ far downstream, and the shock is located at $x=0$. In the following, all the quantities in the upstream region will be indicated with the subscript 1 , and all the quantities downstream with the subscript 2 . To obtain the accelerated proton spectrum affected by energy losses induced by the interaction with the CMBR, we adopt the numerical scheme described in Vannoni et al. (2009). The time-dependent transport equation for the particle distribution function in phase space is

$$
\begin{aligned}
& \frac{\partial f(x, p, t)}{\partial t}+u \frac{\partial f(x, p, t)}{\partial x}-\frac{\partial}{\partial x}\left(D(x, p) \frac{\partial f(x, p, t)}{\partial x}\right) \\
& -\frac{p}{3} \frac{\partial u}{\partial x} \frac{\partial f(x, p, t)}{\partial p}-\frac{1}{p^{2}} \frac{\partial}{\partial p}\left(p^{2} L(x, p) f(x, p, t)\right)=Q(x, p),
\end{aligned}
$$

where $L(x, p)=-\dot{p}$ is the energy loss rate, $Q(x, p)$ the injection term, and $u$ represents the bulk velocity of the plasma in the shock rest frame. The momentum and space dependence of the energy loss term, as well as of the diffusion coefficient, can be chosen of any form so that the formulation is general. 
G. Vannoni et al.: Acceleration and radiation of ultra-high energy protons in galaxy clusters

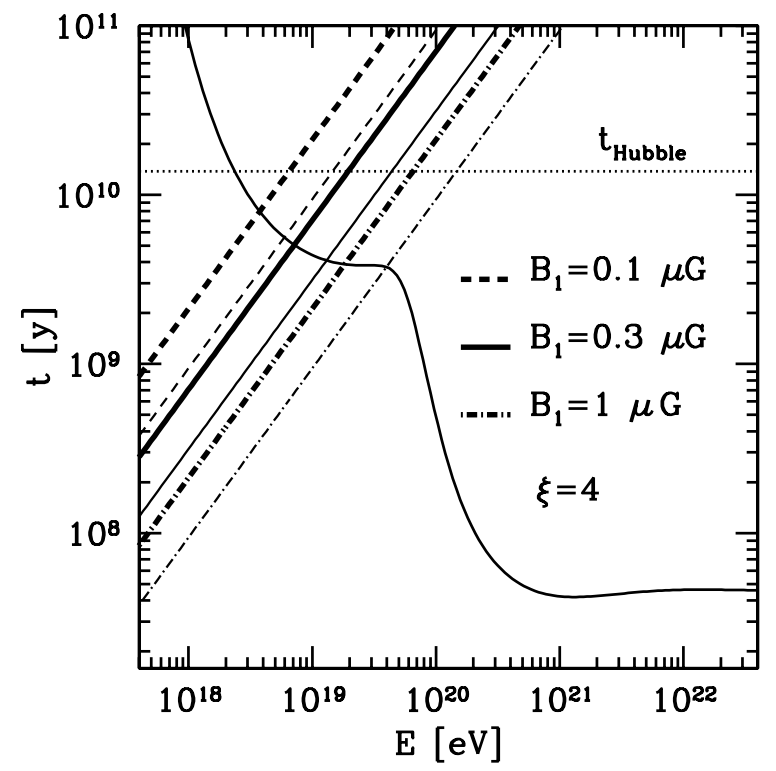

Fig. 1. Acceleration and energy loss time scales as a function of the proton energy. The acceleration time scales are obtained for the values of the upstream magnetic field $B_{1}$ reported in figure and a downstream magnetic field $B_{2}=4 B_{1}$. The thick lines correspond to a shock velocity of $2000 \mathrm{~km} \mathrm{~s}^{-1}$, the thin lines to a velocity of $3000 \mathrm{~km} \mathrm{~s}^{-1}$. The horizontal dotted line shows the estimated age of the Universe, for comparison.

Equation (2) is solved numerically, inserting the boundary conditions at the shock location and at upstream/downstream infinity. For the boundary at the shock we consider again Eq. (2) and integrate it between $x=0_{-}$immediately upstream and $x=0_{+}$ immediately downstream, leading to

$\frac{1}{3}\left(u_{1}-u_{2}\right) p \frac{\partial f_{0}}{\partial p}=\left.D_{2} \frac{\partial f_{0}}{\partial x}\right|_{2}-\left.D_{1} \frac{\partial f_{0}}{\partial x}\right|_{1}+Q_{0} \delta\left(p-p_{0}\right)$.

At $\pm \infty$ we set $f(x, p)=0$.

We assume that injection happens at the shock surface and that it can be described by a delta-function in momentum:

$Q(x, p)=Q_{0} \delta(x) \delta\left(p-p_{0}\right)$,

where $p_{0}$ is the injection momentum and $Q_{0}$ a normalisation constant.

As pointed out in Norman et al. (1995) and Kang et al. (1997), the relevant energy loss channel for the protons accelerated at cluster accretion shocks is the interaction with the CMBR. Other radiation fields do not play any significant role in this regard. The interaction proceeds via two main processes of electron/positron pair production, also known as Bethe-Heitler pair production, and photomeson production. In Fig. 1 we show the cooling time scales of protons due to these two processes. Pair production dominates up to energies of about $5 \times 10^{19} \mathrm{eV}$. Once the particle energy has passed the threshold for meson production, this latter becomes rapidly dominant. The downstream magnetic field is assumed of the form $B_{2}=\xi B_{1}$, where $\xi$ is the compression factor at the shock. For a strong linear shock, the parameter $\xi$ varies between one and four, depending on the orientation of the upstream magnetic field. We choose $\xi=4$, appropriate for Alfvènic turbulence. Figure 1 shows that if protons can be accelerated beyond a few times $10^{18} \mathrm{eV}$, both the cutoff energy and the shape of the particle spectrum in the cut-off region are determined by pair production. Even for extreme cluster parameters, the protons do not achieve energies for which the energy losses would be dominated by photomeson production.

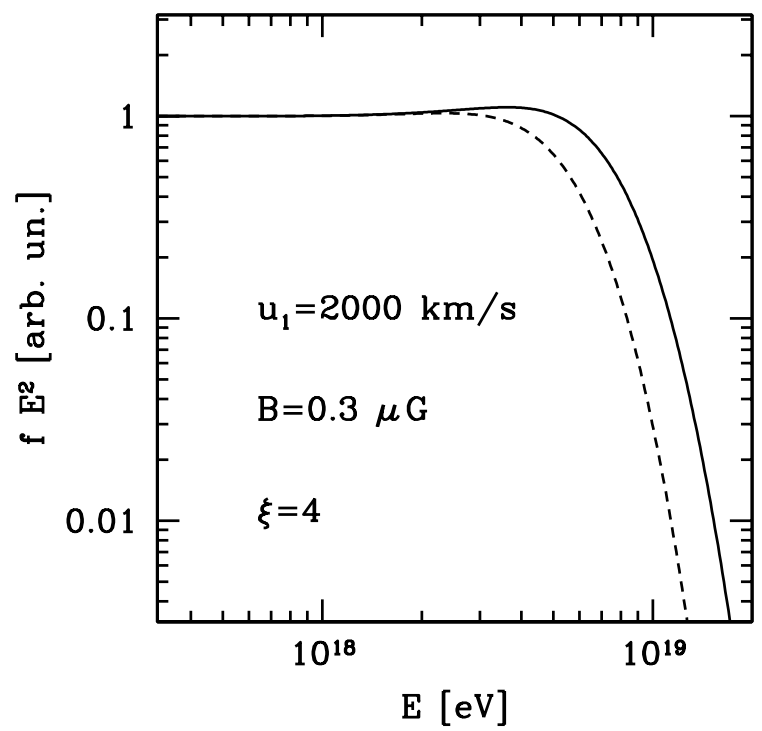

Fig. 2. Proton spectra at the shock location for an acceleration time of $10 \mathrm{Gyr}$ (solid line) and $5 \mathrm{Gyr}$ (dashed) for a shock velocity of $2000 \mathrm{~km} \mathrm{~s}^{-1}$, a magnetic field upstream $B_{1}=0.3 \mu \mathrm{G}$ and a magnetic field downstream $B_{1}=4 B_{1}$.

In this work we have chosen the following values for model parameters: a shock velocity of $2000 \mathrm{~km} \mathrm{~s}^{-1}$ and a magnetic field upstream $B_{1}=0.3 \mu \mathrm{G}$, with $B_{2}=4 B_{1}$. We do not take the evolution of the CMBR with redshift into account. This simplification is justified by the fact that the acceleration rate and the energy loss rate become comparable on a time scale of about 5 Gyr. Therefore, for local clusters, this time scale places the start of the acceleration process at an epoch where the redshift is $z \ll 1$ and we can neglect the evolution of the CMBR. Moreover, as discussed in Kang et al. (1996), the higher temperature and energy density of the CMBR at high redshifts inhibits the acceleration of protons to ultra-high energies at an early epoch.

In Fig. 2 we plot the calculated proton spectra at the shock location. Our results are more conveniently shown as a function of the particle energy, rather than their momentum. We performed our calculation for an age of the system of $5 \mathrm{Gyr}$ and $10 \mathrm{Gyr}$. The difference between the spectra at the two ages demonstrates that the system does not reach steady state. Therefore, a time dependent calculation is essential in order to model the particle spectrum correctly. The cut-off energy is located around $7 \times 10^{18} \mathrm{eV}$. A small bump is present in the spectra around the cut-off energy, due to the flattening of the energy loss time scale in that energy range. The prominence of the feature increases with time.

Once the particle distribution function was obtained for each point of the phase space, we proceeded by calculating the spectrum integrated over space $F(E)=\int f(x, E) \mathrm{d} x$, in order to obtain the production spectra of secondary pairs. The results are plotted in Fig. 3 for the up- and down-stream regions of the shock separately. The spectral features are evident in the spatially integrated spectra. At energies below the cut-off, the upstream particle spectrum is hard. This is because particles can propagate ahead of the shock front over a distance of the same order of their diffusion length, defined as $x_{D}=D(E) / u_{1}$. For Bohm-type diffusion $x_{D} \propto E$, and the integrated spectrum upstream is thus proportional to the particle spectrum at the shock multiplied by $E$. Downstream, on the other hand, at energies low enough that energy losses are negligible, the spectrum is $f_{2}(x, E)=f_{0}(E) \propto E^{-2}$. In this component, a bump forms around $10^{18} \mathrm{eV}$ because around that energy (close to the threshold of the 


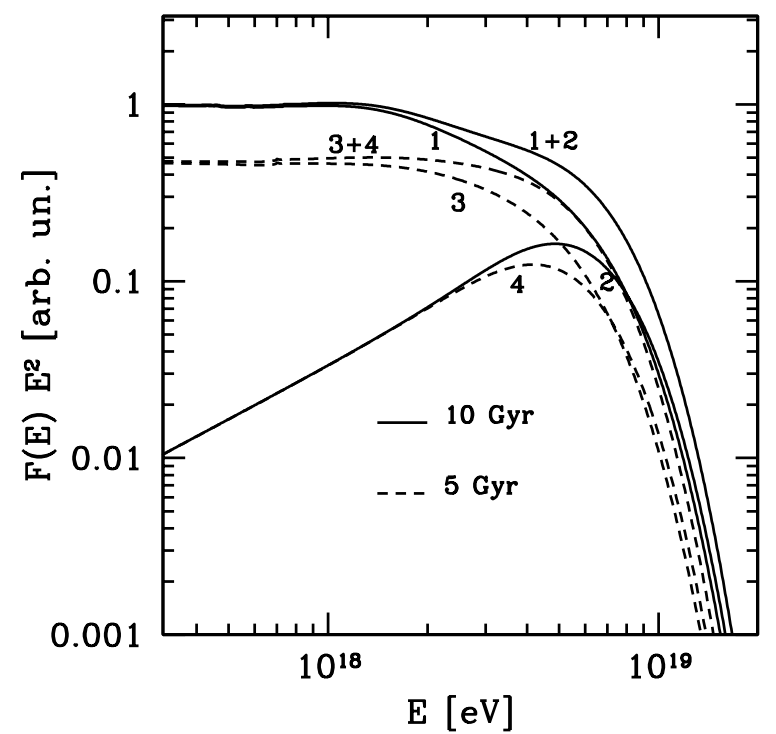

Fig. 3. Spatially integrated spectra for the proton distributions in Fig. 2 for an accelerator age of $10 \mathrm{Gyr}$ (solid lines) and $5 \mathrm{Gyr}$ (dashed lines). The lines 1 and 3 represent the downstream contributions, 2 and 4 the upstream ones, and $1+2$ and $3+4$ the sum of each pair.

process) the pair production loss rate changes behaviour from a very steep dependence on the particle energy to an almost flat distribution. Therefore, particles at slightly lower energies are only marginally affected by losses, as we can see from the fact that, below $10^{18} \mathrm{eV}$, a power law spectrum $\propto E^{-2}$ is recovered, as expected for an uncooled spectrum; on the other hand, particles at slightly higher energies experience a very fast cooling so that there is an effect of accumulation around the threshold. The effect is analogous to the one discussed in Berezinsky \& Grigoreva (1988), explaining the appearance of a bump in the ultra-high energy cosmic ray spectrum due to photomeson production during propagation. Quite interestingly, as a consequence of the shallow dependence on energy of the loss time scale at the cut-off energy and above it, the spectrum in the cut-off region is smoother than a simple exponential behaviour. At energies around $10^{20} \mathrm{eV}$, a second steepening is in fact present (not shown in figure), due to the effect of pion production; however, at that energy the flux is suppressed by several orders of magnitude with respect to the cut-off value, and thus meson production is negligible for changing the proton spectrum.

It is worth noting that, even though the contribution from the downstream region is dominant at low energies, around the cut-off, the contribution of the two components differ only by a factor of few.

\section{Spectra of secondary electrons}

In the following we present the results calculated for a cluster with an age of 5 Gyr. For simplicity we assumed that the magnetic field is homogeneously distributed over the cluster. The formalism proposed by Kelner \& Aharonian (2008) was used for calculating the production spectra of secondary electronpositron pairs. Figure 4 shows the spectra of secondary electrons up and downstream produced by the proton spectra shown in Fig. 3.

Once produced, the electrons (hereafter the term "electrons" implies both electrons and positrons) lose energy during interactions with the CMBR and the ambient magnetic field. The energy loss rates for these two processes are shown in Fig. 5.

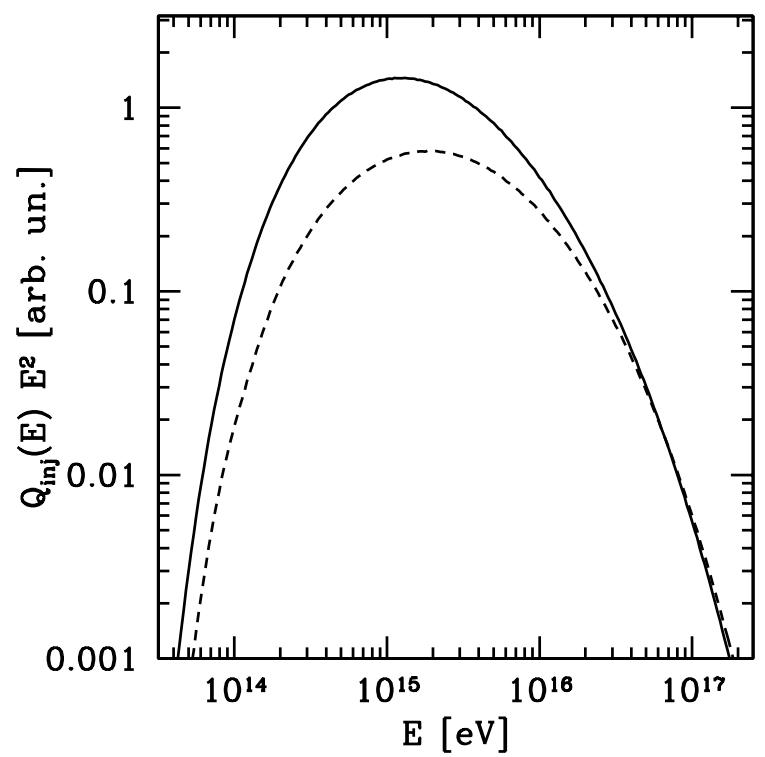

Fig. 4. Production spectra of pairs. Solid line: downstream spectrum for an accelerator age of $5 \mathrm{Gyr}$; dashed line: upstream spectrum.

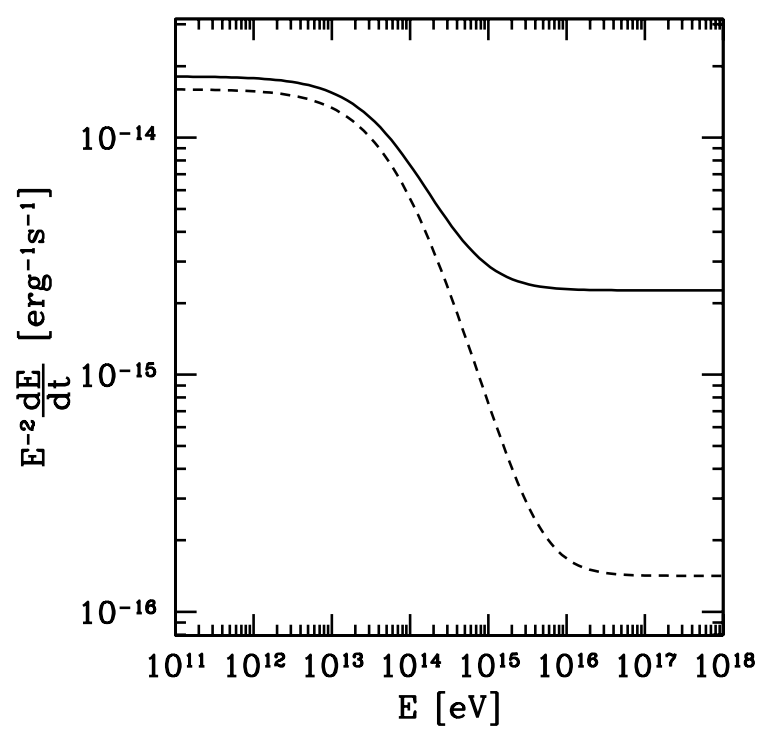

Fig. 5. Energy loss rates for pairs due to synchrotron and IC processes. Solid line: downstream (magnetic field $B_{2}=1.2 \mu \mathrm{G}$ ), dashed line: upstream (magnetic field $B_{1}=0.3 \mu \mathrm{G}$ ).

The curves are multiplied by $E^{-2}$ to highlight the transition of the Compton losses from the Thomson to Klein-Nishina regime, which takes place around $10 \mathrm{TeV}$. The curves correspond to the sum of synchrotron and IC energy loss rates in the downstream and upstream regions. In both cases the target photon field for IC is the CMBR with temperature $2.7 \mathrm{~K}$. The magnetic field downstream is four times larger than upstream. While at low energies the Compton losses are greater than the synchrotron losses, at very high energies, $E \geq 1000 \mathrm{TeV}$, because of the Klein-Nishina effect, the synchrotron losses become the dominant channel for cooling electrons.

For the given injection (source) rate $Q_{\text {inj }}(E)$, the energy distribution of electrons $F(E)$ is described, in the continuous energy-loss approximation, by the equation

$\frac{\partial F(E)}{\partial t}-\frac{\partial}{\partial E}\left(\frac{\mathrm{d} E}{\mathrm{~d} t} F(E)\right)=Q_{\mathrm{inj}}(E)$. 
G. Vannoni et al.: Acceleration and radiation of ultra-high energy protons in galaxy clusters
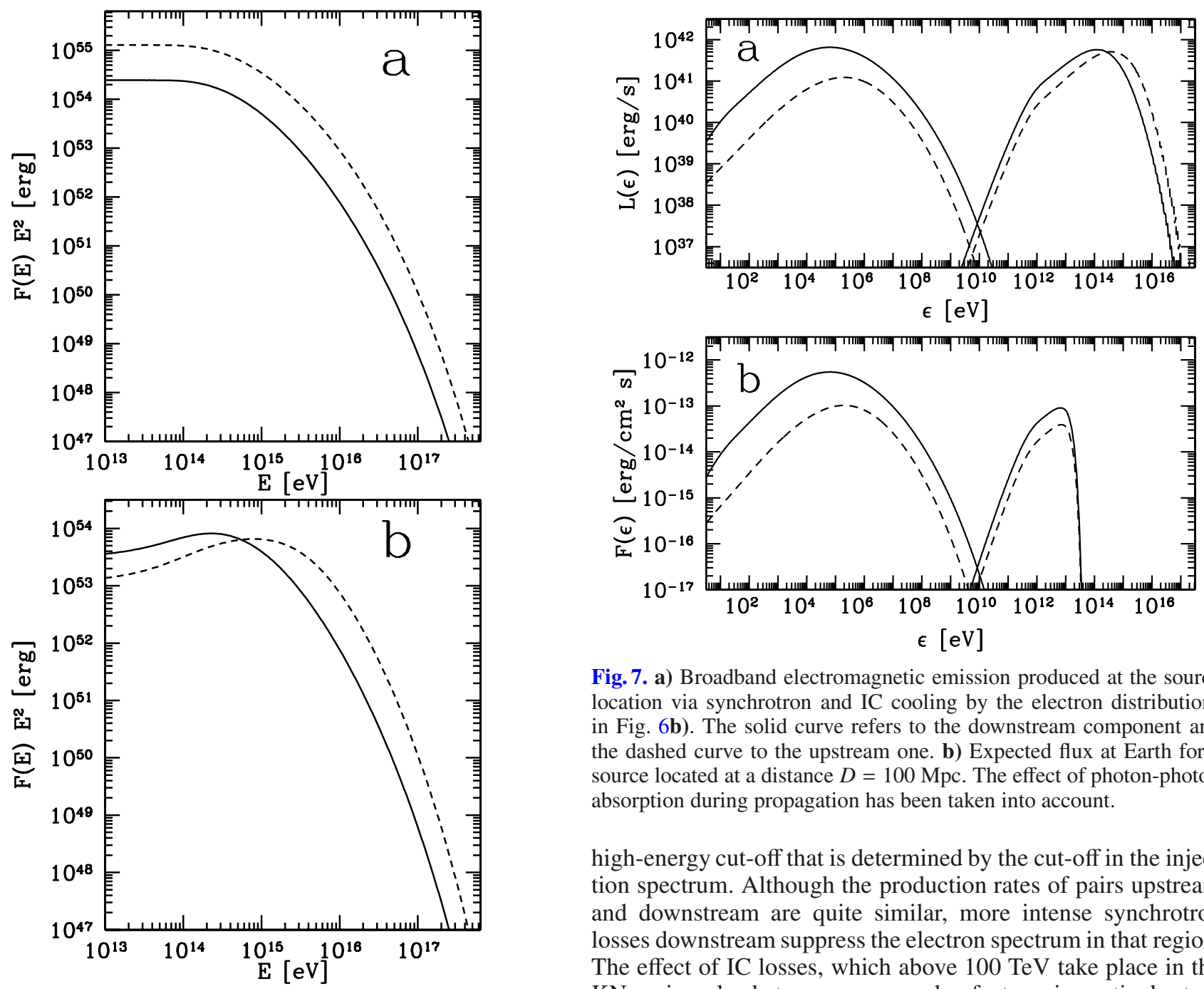

Fig. 7. a) Broadband electromagnetic emission produced at the source location via synchrotron and IC cooling by the electron distributions in Fig. $6 \mathbf{b}$ ). The solid curve refers to the downstream component and the dashed curve to the upstream one. b) Expected flux at Earth for a source located at a distance $D=100 \mathrm{Mpc}$. The effect of photon-photon absorption during propagation has been taken into account.

high-energy cut-off that is determined by the cut-off in the injection spectrum. Although the production rates of pairs upstream and downstream are quite similar, more intense synchrotron losses downstream suppress the electron spectrum in that region. The effect of IC losses, which above $100 \mathrm{TeV}$ take place in the $\mathrm{KN}$ regime, leads to a more complex feature, in particular to a hardening below the cut-off. Since IC losses affect the two sides of the shock in the same way, the slightly higher production rate of pairs downstream results in a higher flux of cooled electrons at energies up to $10^{15} \mathrm{eV}$. At higher energies the synchrotron losses dominate (see Fig. 5), and thus determine the spectral shape of electrons.

\section{Radiation spectra}

Here we assume that the particles do not undergo any further acceleration during their lifetime. In the steady state limit, $\partial F(E) / \partial t=0$, the energy distribution of electrons is given by

$F(E)=\frac{1}{\mathrm{~d} E / \mathrm{d} t} \int_{E}^{\infty} Q_{\mathrm{inj}}\left(E^{\prime}\right) \mathrm{d} E^{\prime}$.

The steady state solution correctly describes the spectrum of electrons, given that the lifetime of high energy electrons is shorter than the age of the source.

The two panels in Fig. 6 show the cooled spectra of electrons downstream (solid) and upstream (dashed). The normalisation is obtained assuming a total energy in accelerated protons of $10^{62} \mathrm{erg}$ in the volume of the cluster. The spectra are calculated with two assumptions: pure synchrotron losses (panel a), and synchrotron plus IC losses (panel b). The case in panel a is not realistic, but it allows us to understand the effects introduced by IC losses. Because of very hard injection spectrum of electrons below $100 \mathrm{TeV}$ (see Fig. 4), the synchrotron losses lead to a standard $E^{-2}$ type steady state spectrum of electrons with a

We can now calculate the emitted radiation spectra based on the electron distributions in Fig 6b. The broadband spectral energy distribution (SED) of photons produced via synchrotron and IC emission is shown in Fig. 7a. Because of the enhanced magnetic field, the synchrotron emission is higher by a factor of $\approx 10$ downstream, as compared to upstream. At the same time, the synchrotron peaks of the SED both in downstream and upstream regions are located around $100 \mathrm{keV}$ (slightly below and above $100 \mathrm{keV}$, respectively). The larger magnetic field downstream is effectively compensated for by the shift in the maximum of the electron distribution upstream to higher energies (see Fig. 6b). Due to the homogeneous distribution of the target photons for the IC scattering $(2.7 \mathrm{~K} \mathrm{CMBR})$, the resulting IC gamma-ray spectra essentially mimic the electron distributions. In particular, the Compton peak in the upstream region is noticeably shifted compared to the position of the Compton peak characterising the downstream region.

Unlike previous calculations of proton spectra in galaxy clusters, we do not assume an a priori spectrum, e.g. of the 
"standard" form $E^{-2} \exp \left[-E / E^{*}\right]$, but instead calculate its shape within the model of DSA, taking energy losses into account. Interestingly, our self-consistent calculation shows a less steep fall off in the final emitted radiation spectrum above the cut-off energy. The effect is the result of the combination of two factors: i) the accurate treatment of DSA correctly taking energy losses into account; ii) the accurate calculation of the shape of the produced electron spectrum. In this regard we note that the commonly used delta-function approximation significantly deviates from the results of accurate calculations (Kelner \& Aharonian 2008).

While some hints of non-thermal hard X-ray emission have been reported from the Coma and Ophiuchus clusters (e.g. Fusco-Femiano et al. 1999; Eckert et al. 2008), these objects have not yet been detected in gamma rays. Our calculations show that, if protons are effectively accelerated by accretion shocks, one should expect both hard X-rays and TeV gamma-rays from nearby and powerful clusters. The detectability of the X-ray and gamma-ray fluxes depend on the total energy of protons accumulated in the cluster over the lifetime of the source (up to $10 \mathrm{Gyr}$ ) and the distance to the source. Also the angular size of the emission is likely to play a role, since nearby clusters of galaxies will appear as quite extended sources. Normalising the total energy of protons in the cluster to $10^{62} \mathrm{erg}$, the luminosities in both synchrotron and IC components of emission are expected at the level of $L \sim 10^{42} \mathrm{erg} / \mathrm{s}$. To estimate the expected flux, $F_{\mathrm{E}}=L / 4 \pi d^{2}$, one has to fix the distance to the object. For example, in the case of the Coma cluster $(d=100 \mathrm{Mpc})$, the results are shown in Fig. 7b, where the effect of gamma-ray photon absorption by the extragalactic background light (EBL) has been taken into account. In the calculation we used the recent EBL flux from Franceschini et al. 2008. The energy flux for the synchrotron component appears to be of the order of

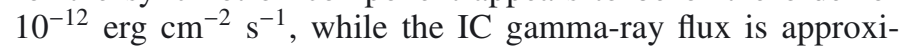
mately one order of magnitude less, given that gamma rays with energy exceeding $10 \mathrm{TeV}$ are effectively absorbed in the EBL. The detection of these fluxes from extended regions such as clusters of galaxies is not an easy task, but possibly feasible with the next generation of hard X-ray and $\mathrm{TeV}$ gamma-ray detectors.

All the results presented above refer to a value of the upstream (downstream) magnetic field equal to $0.3 \mu \mathrm{G}(1.2 \mu \mathrm{G})$. From Fig. 1 one can easily see that the effect of varying the magnetic field at the shock is to shift the maximum energy of accelerated particles. For example, for an upstream field of 0.1 , 0.3 , and $1 \mu \mathrm{G}$, and for a cluster with an age of $5 \mathrm{Gyr}$ and shock speed $2000 \mathrm{~km} \mathrm{~s}^{-1}$, one can infer a value for the maximum energy of accelerated particles of roughly $2 \times 10^{18}, 7 \times 10^{18}$, and $2 \times 10^{19} \mathrm{eV}$, respectively. The resulting spectrum of secondary electron-positron pairs is a broad bell-shaped curve, with maximum roughly at an energy: $\left(m_{\mathrm{e}} / m_{\mathrm{p}}\right) E_{\mathrm{p}}$, where $E_{\mathrm{p}}$ is the parent proton energy. This means that changing the value of the magnetic field at the shock in the range $0.1-1 \mu \mathrm{G}$ will correspondingly shift the peak of the production spectrum of pairs about one order of magnitude. This would imply a shift of (very roughly) the same amount for the peak of the X-ray synchrotron emission. Conversely, for the inverse Compton scattering emission, the peak is not going to change significantly, since it is mainly determined by the absorption in the intergalactic radiation field (see e.g. Fig. 7, bottom panel).

\section{Shock modification}

We have shown in the previous sections that protons can be efficiently accelerated at cluster accretion shocks. In this scenario,

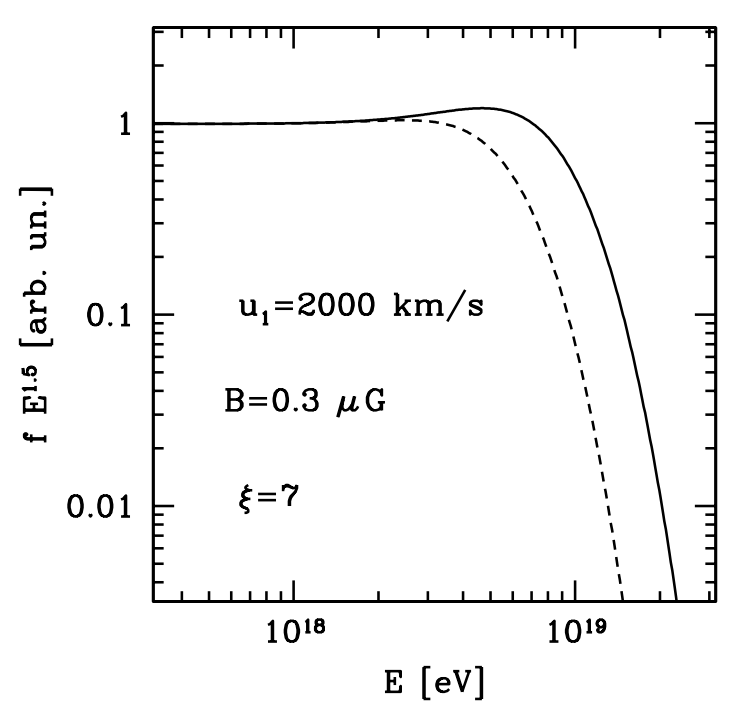

Fig. 8. Proton spectra at the shock location for the case of a shock compression ratio $R=7$. The solid curve refers to an acceleration time of $10 \mathrm{Gyr}$, the dashed curve to an acceleration time of $5 \mathrm{Gyr}$. The shock velocity is $2000 \mathrm{~km} \mathrm{~s}^{-1}$, the magnetic field upstream is $B_{1}=0.3 \mu \mathrm{G}$ and downstream $B_{2}=7 B_{1}$.

the reaction of the accelerated particles on the structure of the shock itself can be quite significant, leading to its modification. In turn, the modification of the shock reflects on the particle spectrum. This complex interplay leads to a variety of non-linear effects. A great deal of study has been dedicated to non-linear shock acceleration in recent years (see Malkov \& Drury 2001 for a review). One of the consequences of shock modification is the increase in the acceleration efficiency coupled with a significant hardening of the energy distribution of protons. As a result, the available kinetic energy transferred to accelerated particles is accumulated at the highest energies close to the cut-off. This part of the spectrum provides the dominant contribution to the pair production, and ultimately to the broad-band electromagnetic radiation emitted by the secondary electrons, therefore the features of the shock modification should be reflected in the spectrum of the non-thermal electromagnetic radiation.

In order to explore this possibility, we consider the modification of the shock in a simplified way, a full non-linear treatment being beyond the scope of the present study. We assume a compression ratio at the shock $R=7$, which results from the linear theory of DSA when the relativistic component of the fluid (i.e. the accelerated particles) dominate the system's dynamics. Analogously, the magnetic compression factor is increased to $\xi=7$. In this case the spectral slope predicted by the theory in the absence of losses is $\alpha=1.5$. The calculated proton spectrum at the shock surface, multiplied by $E^{1.5}$, is shown in Fig. 8 for source ages of 5 and 10 Gyr. One can see in the spectrum, just before the cut-off, a bump that is induced by the energy losses due to interactions with the CMBR.

In Fig. 9 we show the spatially integrated spectra of protons in the upstream and downstream regions. The curves are multiplied by $E^{2}$ to emphasise that the energy transferred to protons is accumulated just before the cut-off energy region.

The secondary pair-production spectra are shown in Fig. 10, while Fig. 11 shows the energy loss rates for electrons upstream and downstream. Compared to Fig. 5, the synchrotron cooling in the downstream region is increased due to the higher magnetic field. This has an impact on the cooled spectra, as shown in 
G. Vannoni et al.: Acceleration and radiation of ultra-high energy protons in galaxy clusters

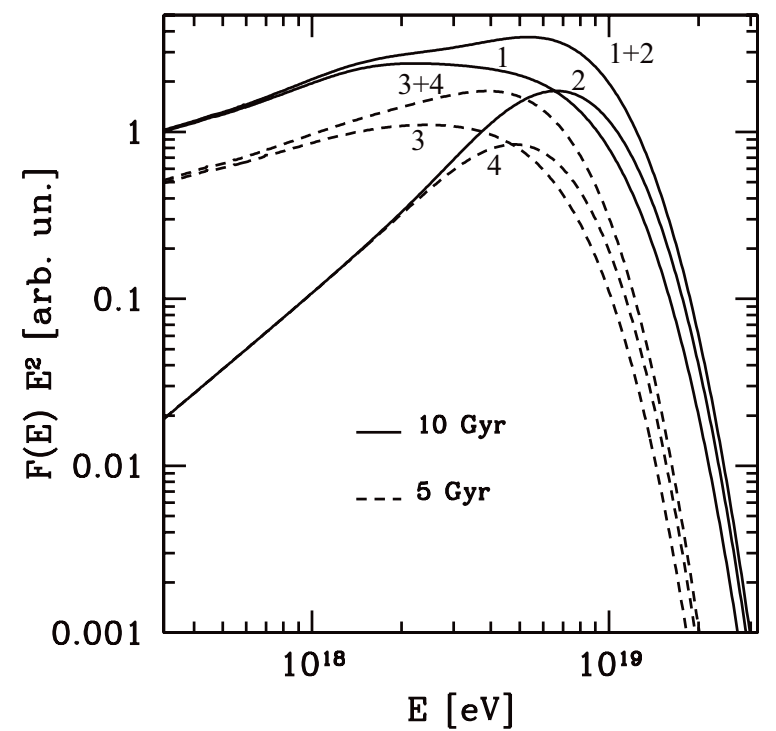

Fig. 9. Spectra integrated over the upstream (2 and 4) and downstream (1 and 3) regions for the proton distributions in Fig. 8. The solid lines correspond to an accelerator age of $10 \mathrm{Gyr}$ and the dashed one to an age of 5 Gyr.

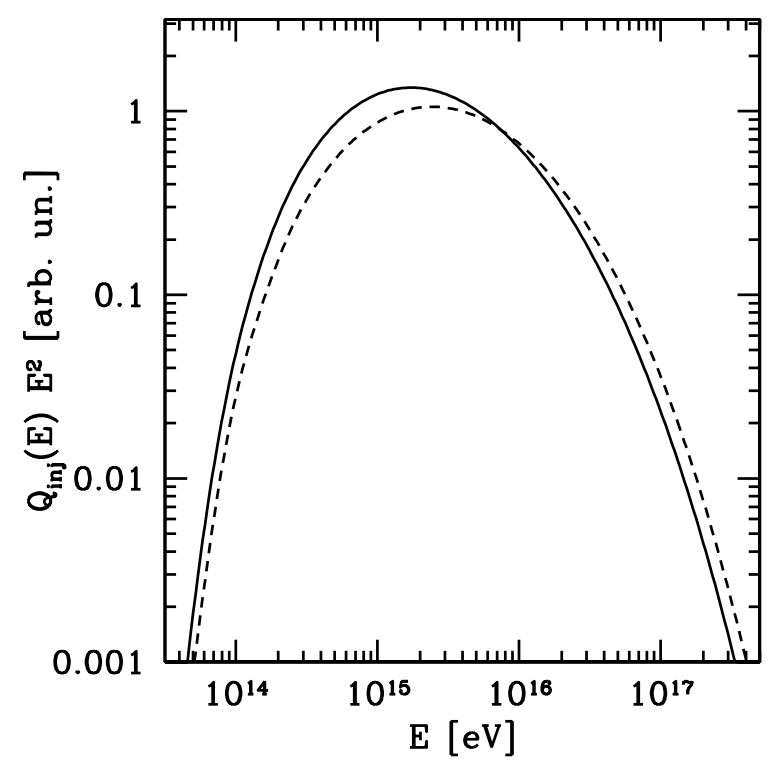

Fig. 10. Pair production spectra for an accelerator age of 5 Gyr. The solid line represents the downstream component, the dashed line refers to the upstream component.

Fig. 12. The spectra are normalised to a total energy released in high-energy protons of $10^{62} \mathrm{erg}$. The spectral characteristics are similar to those shown in Fig. 6, but, due to the hard spectrum of protons, the amount of energy released in pairs is an order of magnitude more than for $R=4$.

This effect is directly reflected in the broadband radiation produced by secondary electrons (Fig. 13a). The X-ray luminosity is increased by an order of magnitude compared to the case of $R=4$. The same applies for the IC radiation emitted in the upstream region.

Figure $13 \mathrm{~b}$ shows the expected photon fluxes at Earth for a source located at a distance $d=100 \mathrm{Mpc}$. Because of the intergalactic absorption, the gamma-ray flux above $10 \mathrm{TeV}$ is dramatically suppressed. This leads to the shift in the maximum

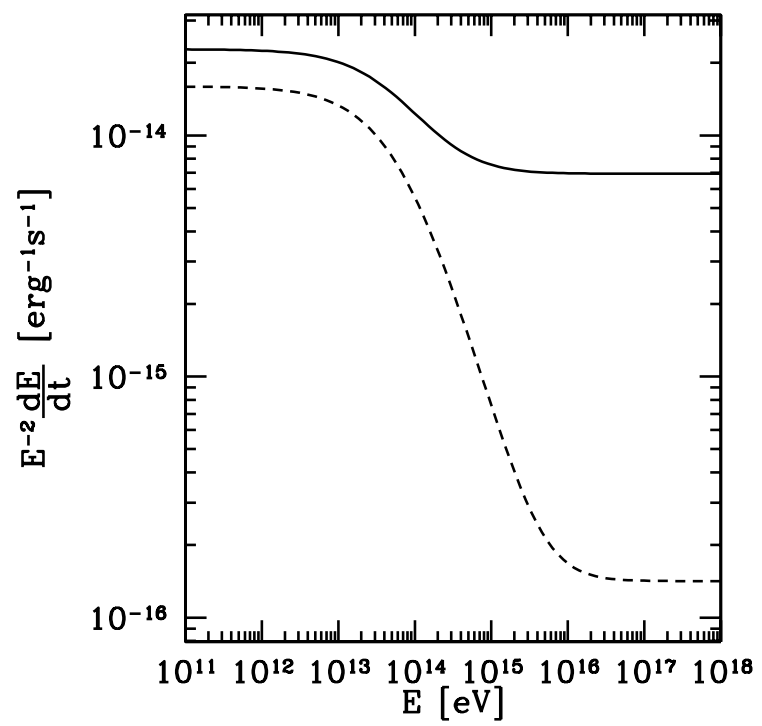

Fig. 11. Energy loss rates for synchrotron plus IC losses. The magnetic field upstream is $B_{1}=0.3 \mu \mathrm{G}$ (dashed line), in the downstream region $B_{2}=7 B_{1}$ (solid line).

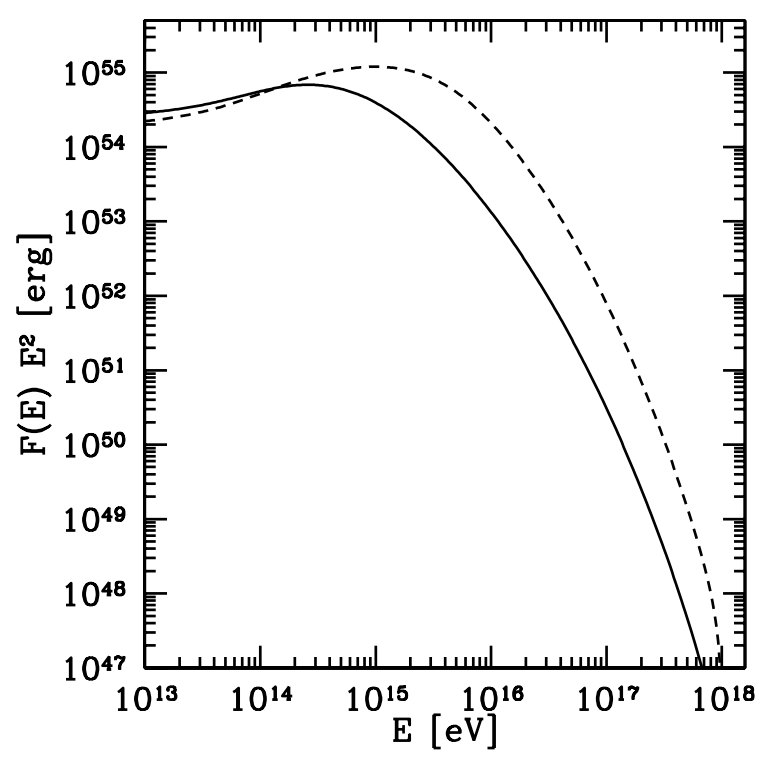

Fig. 12. Electron spectra after cooling, downstream (solid line) and upstream (dashed line), obtained by considering the energy losses in Fig. 11.

of the arriving IC gamma-ray spectrum to $10 \mathrm{TeV}$ with a flux at the level of $10^{-12} \mathrm{erg} / \mathrm{cm}^{2} \mathrm{~s}$.

We remind here that the choice of a power law with index 1.5 for the proton spectrum constitutes an extreme case, given that the slope 1.5 is the hardest slope allowed by non-linear diffusive shock acceleration. Thus, the proton spectra calculated in the linear regime (i.e. the ones with slope $E^{-2}$ below the high-energy cutoff shown in Sect. 2), and the ones shown in this section represent the two extreme cases of unmodified and strongly modified shock. Therefore, we can conclude that, if we fix the total energy converted into accelerated particles, the effect of shock modification can increase the resulting radiation flux up to an order of magnitude if compared with the test particle case. 

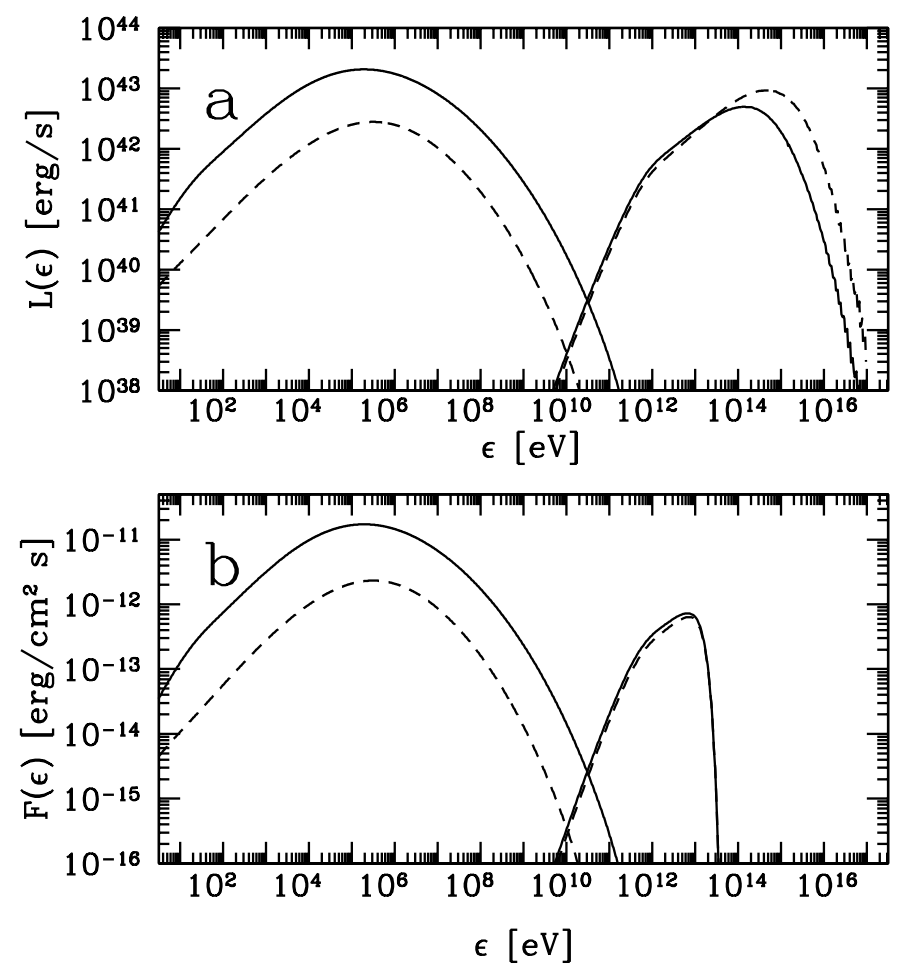

Fig. 13. a) Broadband radiation spectra produced at the source by the electron distributions in Fig. 12 b), downstream (solid line) and upstream (dashed line). b) Energy flux at the observer location, after absorption in the EBL, for a source distance of $100 \mathrm{Mpc}$.

\section{Conclusions}

Proton acceleration in galaxy clusters was studied in the framework of DSA via a detailed time-dependent numerical calculation that includes energy losses due to interactions of protons with photons of the CMBR. For realistic shock speeds of a few thousand $\mathrm{km} \mathrm{s}^{-1}$ and a background magnetic field close to $1 \mu \mathrm{G}$, the maximum energy achievable by protons is determined by the energy losses due to pair production and ranges from a few times $10^{18} \mathrm{eV}$ to a few times $10^{19} \mathrm{eV}$.

We performed the calculations assuming that acceleration takes place on time scales comparable to the age of the cluster. Since steady state is never achieved in this scenario, a timedependent treatment is required. When calculated including the effect of energy losses, particle spectra exhibit interesting features. The decay of the spectrum above the cut-off energy is not exponential. Its dependence on energy is shallower due to the flat profile of pair production timescales in the cut-off energy range. The time-dependent distributions of protons are used to accurately calculate the production rates of secondary electronpositron pairs. These electrons cool rapidly via synchrotron radiation and IC scattering that proceeds in the Klein-Nishina regime. The effect of the hardening induced by the KN crosssection is visible in the IC radiation spectra both in the upstream and downstream regions of the shock. For the fiducial Coma-like cluster used in this work, the synchrotron and IC peaks of the electron broadband SED are at comparable levels and the associated flux from a source at the distance of a $100 \mathrm{Mpc}$ is expected at the level of $10^{-12} \mathrm{erg} \mathrm{cm}^{-2} \mathrm{~s}^{-1}$ in the X-rays and an order of magnitude lower for $\mathrm{TeV}$ gamma rays. Although the maximum of the gamma-ray emission is located above $100 \mathrm{TeV}$, it unfortunately cannot be observed because of severe intergalactic absorption. The expected gamma-ray flux from clusters of galaxies is at the limit of the sensitivity of present generation instruments, however it may be detectable with the future generation of detectors. The optimum energy interval for gamma-ray detection is between 1 and $10 \mathrm{TeV}$.

The detectability of clusters in hard X-rays and gamma-rays associated with interactions of ultra-high energy protons with the CMBR, depends on the value of the parameter $A=W_{62} / d_{100}^{2}$, where $W_{62}=W / 10^{62} \mathrm{erg}$ is the total energy released in cosmic rays normalised to $10^{62} \mathrm{erg}$, and $d_{100}=d / 100 \mathrm{Mpc}$ is the distance normalised to $100 \mathrm{Mpc}$. Obviously, the best candidates for detection are nearby rich galaxy clusters like Coma and Perseus located at distances $d \sim 100 \mathrm{Mpc}$. On the other hand, owing to the large extension of the non-thermal emission (as large as several $\mathrm{Mpc}$ ), and given that the angular size of the source $\theta \propto 1 / d$, the probability of detection reduces with the distance slower than $1 / d^{2}$. Nevertheless, as long as the total energy in accelerated protons does not significantly exceed $10^{62} \mathrm{erg}$, the visibility of clusters of galaxies in X-rays and gamma-rays is limited by objects located within a few $100 \mathrm{Mpc}$.

The chances of detection of non-thermal emission of clusters related to ultra-high energy protons, especially in the hard X-ray band, can be significantly higher if protons are accelerated by non-linear shocks modified by the pressure of relativistic particles. In this scenario, a large fraction of the energy of the shock is transferred to relativistic protons. Moreover, in this case a very hard spectrum of protons is formed, thus the main fraction of non-thermal energy is carried by the highest energy particles. These two factors can enhance the luminosity of X- and gammaray emission of secondary electrons by more than an order of magnitude, and thus increase the probability of detecting clusters located beyond $100 \mathrm{Mpc}$.

In this paper we did not discuss the gamma-ray production related to interactions of accelerated protons with the ambient gas that can compete with the inverse Compton radiation of pair produced electrons. The relative contributions of these two channels depend on the density of the ambient gas and the spectral shape of accelerated protons. The flux of gamma rays from $p p$ interactions can be easily estimated from the cooling time of protons, $t_{\mathrm{pp}} \approx 1.5 \times 10^{19} / n_{-4} \mathrm{~s}$, where $n_{-4}=n / 10^{-4} \mathrm{~cm}^{-3}$ is the density of the ambient hydrogen gas, normalised to $10^{-4} \mathrm{~cm}^{-3}$. Then, the energy flux of gamma rays at $1 \mathrm{TeV}$ is estimated as $F_{\gamma}(\sim 1 \mathrm{TeV}) \approx 6 \times 10^{-12} \kappa \mathrm{W}_{62} \mathrm{n}_{-4} / \mathrm{d}_{100} \operatorname{erg~cm}{ }^{-2} \mathrm{~s}^{-1}$, where $\kappa$ is the fraction of the total energy of accelerated protons in the energy interval between 10 and $100 \mathrm{TeV}$. These protons are primarily responsible for production of gamma-rays of energy $\sim 1 \mathrm{TeV}$. For a proton energy spectrum extending to $10^{18} \mathrm{eV}$, this fraction is $\kappa \sim 0.1$. Thus for an average gas density in a cluster like Coma, $n \sim 3 \times 10^{-4} \mathrm{~cm}^{-3}$, the gamma-ray flux at $1 \mathrm{TeV}$ is expected at the level of $10^{-12} \mathrm{erg} \mathrm{cm}^{-2} \mathrm{~s}^{-1}$ which is comparable to the contribution of IC radiation of secondary electrons. In the case of harder spectra of protons accelerated by non-linear shocks, the contribution of gamma rays from $p p$ interactions is dramatically reduced and the contribution of secondary pairs to gamma rays via IC scattering strongly dominates gamma rays from $p p$ interactions.

To conclude, we comment on recent results from the Auger experiment, which seem to indicate that the composition of ultra high energy cosmic rays becomes richer in heavier elements at higher energies $\left(\approx 10^{19.5} \mathrm{eV}\right.$ ) (Abraham et al. 2010). One may wonder whether clusters of galaxies might be potential sources of those ultra-high energy cosmic rays. If we consider, as in Fig. 1, a shock speed of $2000 \mathrm{~km} \mathrm{~s}^{-1}$ and a magnetic field 
upstream of the shock of $1 \mu \mathrm{G}$ we found, for iron nuclei, an acceleration time of $t_{\mathrm{acc}} \approx 0.8\left(E / 10^{20} \mathrm{eV}\right)$ Gyr. The main loss channel for nuclei is photo-disintegration in the cosmic microwave background and in the infrared background. By comparing the acceleration time with the energy loss time, we obtain that the maximum energy of accelerated iron nuclei is roughly $\approx 10^{20} \mathrm{eV}$, the exact figure slightly depending on the model adopted to describe the cosmic infrared background (see e.g. Allard \& Protheroe 2009). The same result has been obtained by Inoue et al. (2007). Thus, it seems that the mechanism of shock acceleration operating at accretion shocks around massive clusters of galaxies might possibly contribute to the flux of observed cosmic rays at the highest energies.

Acknowledgements. We would like to thank O. Zacharopoulou and A. M. Taylor for fruitful discussions. G.V. acknowledges support from the International MaxPlanck Research School (IMPRS) Heidelberg. S.G. acknowledges the support of the European Community under a Marie Curie Intra-European fellowship.

\section{References}

Abraham, J., Abreu, P., Aglietta, M., et al. 2010, Phys. Rev. Lett., 104, 091101 Aharonian, F. A. 2002, MNRAS, 332, 215

Aharonian, F. A., Akhperjanian, A. G., Anton, G., et al. 2009, A\&A, 502, 437 Aleksic, J. (MAGIC Coll.), Pfrommer, C., Pinzke, A., et al. 2010, ApJ, 710, 634 Allard, D., \& Protheroe, R. J. 2009, A\&A, 502, 803

Atoyan, A. M., \& Völk, H. J. 2000, ApJ, 535, 45

Berezinsky, V. S., \& Grigoreva, S. I. 1988, A\&A, 199, 1

Berezinsky, V., Blasi, P., \& Ptuskin, V. S. 1997, ApJ, 487, 529

Berrington, R. C., \& Dermer, C. D. 2003, 594, 709

Bertschinger, E. 1985, ApJS, 58, 39

Blasi, P. 2001, Astrop. Phys., 15, 223

Blasi, P., \& Colafrancesco, S. 1999, Astrop. Phys. 12, 169

Blasi, P., Gabici, S., \& Brunetti, G. 2007, Int. J. Mod. Phys. A, 22, 681

Borgani, S., \& Guzzo, L. 2001, Nature, 409, 39
Brunetti, G., Setti, G., Feretti, L., \& Giovannini, G. 2001, MNRAS, 320, 365

Carilli, C. L., \& Taylor, G. B. 2002, ApJ, 577, 22

Clarke, T. E., Kronberg, P. P., \& Böhringer, H. 2001, ApJ, 547, L111

Dennison, B. 1980, ApJ, 239, 93

Dolag, K., \& Enßlin, T. A. 2000, A\&A, 362, 151

Eckert, D., Produit, N., Paltani, S., Neronov, A., \& Courvoisier, T. J.-L. 2008, A\&A, 479, 27

Franceschini, A., Rodighiero, G., \& Vaccari, M. 2008, A\&A, 487, 837

Fusco-Femiano, R., dal Fiume, D., Feretti, L., et al. 1999, ApJ, 513, 21

Gabici, S., \& Blasi, P. 2003, ApJ, 583, 695

Govoni, F., \& Feretti, L. 2004, Int. J. Mod. Phys. D, 13, 1549

Hillas, A. M. 1984, ARA\&A, 22, 425

Inoue, S., Aharonian, F. A., \& Sugiyama, N. 2005, ApJ, 628, 9

Inoue, S., Sigl, G., Miniati, F., \& Armengaud, E. 2007, Proc. 30th International Cosmic Ray Conference, 4 (HE part 1), 555

Kang, H., Cen, R., Ostriker, J. P., \& Ryu, D. 1994, ApJ, 428, 1

Kang, H., Ryu, D., \& Jones, T. W. 1996, ApJ, 456, 422

Kang, H., Rachen, J. P., \& Biermann, P. L. 1997, MNRAS, 286, 257

Kelner, S. R., \& Aharonian, F. A. 2008, Phys. Rev. D, 78, 4013

Kushnir, D., Katz, B., \& Waxman, E. 2009, J. Cosmol. Atrop. Phys., 9, 24

Loeb, A., \& Waxman, E. 2000, Nature, 405, 156

Malkov, M. A., \& Drury L.O'C. 2001, Rep. Prog. Phys., 64, 429

Miniati, F., Ryu, D., Kang, H., \& Jones, T. W. 2001, ApJ, 559, 59

Newman, W. I., Newman, A. L., \& Rephaeli, Y. 2002, ApJ, 575, 755

Norman, C. A., Melrose, D. B., \& Achterberg, A. 1995, ApJ, 454, 60

Ostrowski, M., \& Siemieniec-Ozieblo, G. 2002, A\&A, 386, 829

Perkins, J. S., Badran, H. M., Blaylock, G., et al. 2006, ApJ, 644, 148

Petrosian, V. 2001, ApJ, 557, 560

Pfrommer, C., Springel, V., Ensslin, T. A., \& Jubelgas, M. 2006, MNRAS, 367, 113

Ryu, D., Kang, H., Hallman, E., \& Jones, T. W. 2003, ApJ, 593, 599

Sarazin, C. L. 1988, X-ray Emission from Clusters of Galaxies (Cambridge University Press), I-X, 1

Sarazin, C. L. 1999, ApJ, 520, 529

Schlickeiser, R., Sievers, A., \& Thiemann, H. 1987, A\&A, 182, 21

Timokhin, A. N., Aharonian F. A., \& Neronov, A.Yu. 2004, A\&A, 417, 391

Tribble, P. C. 1993, MNRAS, 263, 31

Vannoni, G., Gabici, S., \& Aharonian, F. A. 2009, A\&A, 497, 17

Völk, H. J., Aharonian, F. A., \& Breitschwerdt, D. 1996, Space Sci. Rev., 75, 279 